\title{
The Characterization of Pseudomonas fluorescens with the Aid of an Electronic Computer
}

\author{
BY MURIEL E. RHODES \\ Department of Microbiology, University of Reading
}

(Received 27 June 1960)

\begin{abstract}
SUMMARY
Data which had been used to characterize the species $P$ seudomonas fluorescens (Rhodes, 1959), as distinct from the data used to define the genus Pseudomonas, have now been used to obtain an assessment of the over-all similarity of $\mathbf{1 6 9}$ isolates of polarly flagellate bacteria (mainly of the $\boldsymbol{P}$. fuorescens species-group) by means of an electronic computer. The results of this analysis are compared with the arrangement of isolates previously favoured by the author. It was found that the two arrangements were often closely similar; the discrepancies are discussed. The two aeromonads of the collection were picked out by the computer, which also satisfactorily replaced an accidentally misplaced pseudomonad isolate. One hundred and thirty-four isolates previously considered to belong to the $\boldsymbol{P}$. fluorescens species-group were positioned together by the computer in a group with more than $\mathbf{8 0 . 0} \%$ over-all similarity throughout. This confirmed previous conclusions about the validity of this taxonomic unit, even though the description of it may appear imprecise because each character was not possessed by every isolate. The possibility of further species differentiation within this group is discussed, particularly with regard to $\boldsymbol{P}$. aeruginosa. The results suggest that $\boldsymbol{P}$. aeruginosa may be regarded as a variety of $\boldsymbol{P}$. fluorescens. Other evidence was obtained which suggests that further work on the phytopathogenic pseudomonads may well reveal significant differences from $\boldsymbol{P}$. fluorescens. Factors governing the choice of a type strain or culture are discussed.
\end{abstract}

\section{INTRODUCTION}

A study of 169 isolates of polarly flagellate bacteria was described by Rhodes (1959), and suggestions were made for a revised definition of the genus Pseudomonas on the basis of 26 cytological, cultural and biochemical characters common to $\mathbf{1 6 5}$ of the isolates. A further 43 characters were not common to these 165 isolates, and the author discussed whether these characters might be used to define subgroups (? species), although it was evident that very few of the isolates were identical with respect to all these 43 characters. From the examination of this body of data, many attempts were made to discover any subgroups of related isolates. Certain of the characters (e.g. gelatin digestion, milk digestion, nitrate reduction, aesculin hydrolysis, egg-yolk reaction, ability to grow at $5^{\circ}$ ) which have been considered to be useful criteria for distinguishing species in Pseudomonas and closely related genera (e.g. Chromobacterium; Sneath, 1956) were selected as possible differentiating criteria. It was found that there was no complete correlation between any of the 43 characters under consideration (nos. 1-43 listed in Fig. 1). The resultant sub- 


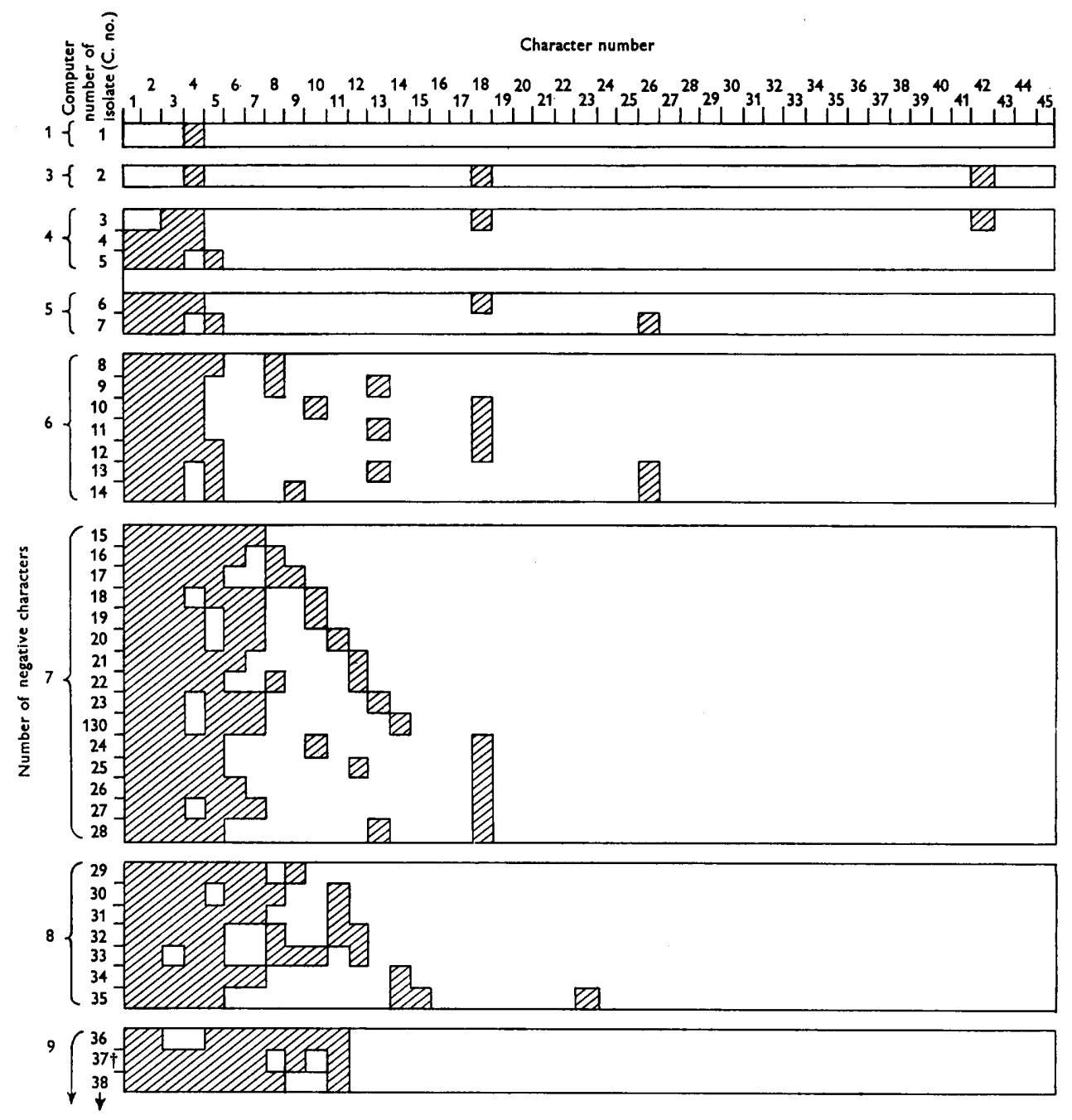

Fig. 1. The properties of the 39 most reactive isolates of Pseudomonas and their arrangement by the author in a table constructed to demonstrate over-all similarity according to the numerical principles described in the text. Hatched areas indicate negative test results; clear areas indicate positive test results. $\dagger=$ proposed neotype strain of Pseudomonas fluorescens (NCTC no. 10,038; ATCC no. 13,525) selected by Rhodes (1959). Key to character numbers : $1=\mathrm{H}_{2} \mathrm{~S}$ from Kligler's medium; $2=$ growth at $42^{\circ} ; 3=\mathrm{H}_{2} \mathrm{~S}$ from cystine; $4=$ aesculin hydrolysis; $5=$ growth in $6.5 \% \mathrm{NaCl}$ broth; $6=$ nitrite reduction; $7=$ nitrate reduction; $8=$ growth from raffinose-C; $9=$ growth in minus-C control; $10=$ growth in minus-N control; $11=$ growth from urea-N; $12=$ growth from sucrose-C; $13=$ hydrolysis of 'Tween 80 '; $14=$ milk digestion; $15=$ egg-yolk reaction; $16=$ olive oil hydrolysis; $17=$ growth from inositol-C; $18=\mathrm{H}_{2} \mathrm{~S}$ from thiosulphate; 19 = growth from cellobiose-C; $20=$ tributyrin hydrolysis; $21=$ gluconic acid oxidation; $22=$ growth from trehalose-C; $23=$ gelatin liquefaction; $24=$ growth from rhamnose-C; $25=$ growth in bile-salt broth; $26=$ growth from $\mathrm{D}$-arabinose-C; $27=$ growth in $5.0 \% \mathrm{NaCl}$ broth; $28=$ growth from succinate-C; $29=$ growth from acetate-C; 30 = growth from mannitol-C; $31=$ growth from lactic acid-C; 32 = growth from lactose-C; $33=$ growth from glycerol-C; $34=$ growth from xylose-C; $35=$ growth from formate-C; 36 = growth from $\mathrm{L}$-arabinose-C; 37 = growth from maltose-C; $38=$ growth at $\mathrm{pH} 5 \cdot 0 ; 39=$ growth from glucose-C; $40=$ growth from galactose-C; $41=$ growth at $5^{\circ} ; 42=$ growth from fructose-C; $43=$ growth from L-malic acid-C; 44 $=$ growth from citrate-C; $45=$ growth from sorbitol-C. 
groups had no other characters in common and therefore were not considered to be useful taxonomic units. The fact that some of these characters had also been shown sometimes to be unstable supported this decision. Three interpretations of this situation were considered. (a) Among the 165 isolates, 39 were replicates; the remaining 126 isolates might be representatives of 126 different subgroups, each differing in at least one of the 43 characters. (b) Perhaps all the 165 isolates belonged to one group with 26 characters in common, with a further 43 characters which might be either present or absent. To adopt this interpretation would be controversial, at least because of the inclusion of about 20 named phytopathogenic species as well as Pseudomonas aeruginosa in this grouping. (c) Perhaps further subdivisions were possible only on the basis of an estimate of the over-all resemblance of the isolates when compared with each other with reference to these 43 characters.

The taxonomic method of 'classification from below' (White, 1937) involves the study of isolates as fully as possible, estimation of the degree of similarity between them, and a final arrangement of the isolates so that similar ones are closely grouped, a greater separation of the isolates (or groups of very similar isolates) being directly related to the magnitude of their dissimilarity. This method was discussed and preferred by Rhodes $(1956,1959)$ as also was the final adoption of the Adansonian principle of according an equal weight to each character studied. The author constructed a table on the basis of a numerical estimate of the over-all similarity of the 165 isolates. This was done by counting the number of positive or negative test results obtained for each of the 43 characters studied. The isolates were then arranged in a sequence such that the first isolate was the most reactive (with $42 / 43$ positive test characters), and the last isolate was the least reactive (only $4 / 43$ positive test characters). The other isolates were then arranged between these extremes (2nd to 164th positions) in order of gradually decreasing reactivity. For example, the 2nd, 3rd and 4th places in the sequence were occupied by isolates of which each had 40 positive test characters; the 5th, 6th and 7 th places, 39; the 8 th, 9 th and 10th places, 38; etc. It became obvious that this ordering of isolates could be split up into subgroups in which each isolate of a given subgroup had the same number of positive characters. However, such subgroups were not found to be useful taxonomic units, because seldom were the same characters negative in any one subgroup (see Fig. 1). Therefore an attempt was made to order the isolates within each of the subgroups. For this, each character was considered separately. In the collection as a whole the frequency of the possession of a positive reaction for each character was useful information, and could be used to determine the order of the isolates in each subgroup in the following way. The \% of all isolates which showed a positive test result for a given character was calculated for each character. The characters were then arranged in an order of increasing \% value. The characters were then numbered serially (1-43); this was the character order number (in brief, character number). For example:

$$
\begin{array}{llll}
\text { Character number: } & 1,2,3,4, \text { to } 43 \\
\text { Isolates positive (\%): } & 1,4,12,17, \text { to } 99
\end{array}
$$

The final order of the isolates in each subgroup was then determined on the principle of according priority within each subgroup to those characters which were more 
frequently negative. For example, subgroup $\mathrm{E}$ might contain 3 isolates each with 4 negative characters as follows:

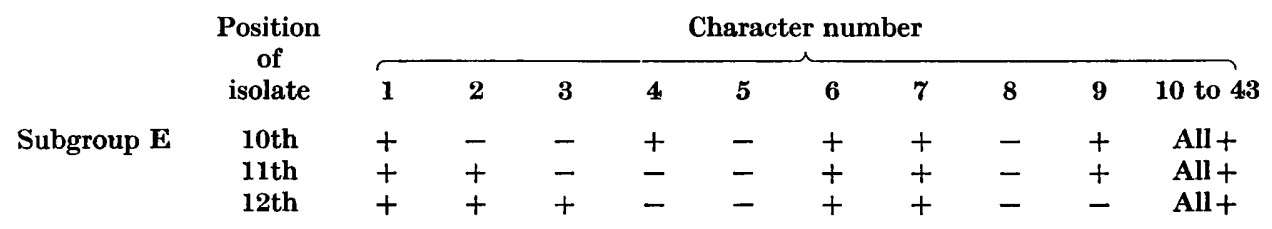

The final order of isolates in the above example was chosen to be 10, 11, 12 because, when the frequency of positive reactions in the collection as a whole was considered, the characters 2 and 3 were more frequently negative than characters 4 and 5 . In this way isolates with negative reactions which were relatively more common were given a smaller ordinal number in the sequence than those with negative reactions which were less common. This minimized the weight of a given negative reaction the more frequently it occurred. This was a deviation from the Adansonian principle of according equal weight to each character, because an isolate with a more unusual negative character would be placed in a relatively later position in each subgroup than if the given character were not so weighted. This arrangement was the culmination of many attempts to give an equal taxonomic weight to each test character. The full table cannot be reproduced here; part of it is given in Fig. 1 and shows how strains may be compared and their similarity illustrated. Fig. 1 also shows the overlapping relationships between isolates.

It appeared that the $\mathbf{1 6 5}$ isolates arranged as just discussed formed a closely related series which showed gradually decreasing reactivity when expressed on the basis of numbers of positive characters. No clear divisions were apparent, but many phytopathogens were concentrated at the 'less-reactive' end of the table and $P$ seudomonas aeruginosa isolates were at the 'reactive' end.

It was clear that the above table contained a body of data suitable for analysis by means of an electronic computer as used by Sneath $(1957 a, b)$ to estimate the over-all similarity on Adansonian principles. With the kind assistance of Dr P. H. A. Sneath the complete set of data was analysed by Elliott Brothers Ltd., by use of the Elliott 405 digital computer and the programme 'Bacterial Classification Mark I' as outlined by Sneath (1957 b). Only 130 isolates were analysed, but these represented the whole collection (including 4 non-Pseudomonas isolates) because the remaining 39 isolates were duplicates of some of the 130 analysed. Table 1 lists the code numbers of the isolates as given by Rhodes (1959) together with the new code numbers needed by the computer. For comparative purposes the position of the $\mathbf{1 6 5}$ isolates in the author's complete table mentioned above (used in Rhodes, 1959) is also included. For the computation 45 characters were used, namely the 43 characters already discussed and named in Fig. 1, together with the results for citrate and sorbitol utilization. The scoring of the features for the computer was simplified because, for theoretical reasons, no quantitative distinctions were made; each character tested for was scored as either positive or negative.

A 'non-coincidental triangle' table (S table) of similarity (S) values for each pair of isolates with respect to the $\mathbf{4 5}$ features was obtained after electronic computation, according to the formula

$$
\mathrm{S}=\frac{n_{s}}{n_{s}+n_{d}}
$$


Table 1. (a) The code numbers of the Pseudomonas isolates studied by Rhodes (1959); (b) the position of the isolates in the author's table constructed to show the relationships between the 169 isolates when they were compared with respect to 45 test characters (see text for details); (c) the new computer code number (C. no.)

Parentheses around numbers indicate that the isolates enclosed in those positions had identical properties, and so only one new computer code number was necessary. $(\dagger)=$ proposed neotype strain $P$ seudomonas fluorescens (Rhodes, 1959); $\left(^{*}\right)=$ NCTC $P$. fluorescens; $(\mathrm{C})=P$. chlororaphis; $(\mathrm{A})=P$. aeruginosa; $(\mathrm{S})=P$. syncyanea $;(\mathrm{P})=$ phytopathogenic Pseudomonas species; $(\mathrm{Ae})=$ Aeromonas; $(\mathrm{X})=\mathrm{Xantho-}$ monas; $(\mathrm{N})=$ non-pseudomonad phytopathogen.

\begin{tabular}{|c|c|c|c|c|c|}
\hline Isolate code number & $\begin{array}{l}\text { Position in } \\
\text { author's } \\
\text { complete } \\
\text { table }\end{array}$ & $\begin{array}{l}\text { Computer } \\
\text { number } \\
\text { (C. no.) }\end{array}$ & Isolate code number & $\begin{array}{l}\text { Position in } \\
\text { author's } \\
\text { complete } \\
\text { table }\end{array}$ & $\begin{array}{c}\text { Computer } \\
\text { number } \\
\text { (C. no.) }\end{array}$ \\
\hline $5940\left(^{*}\right)$ & 1 st & 1 & $25 / 1 ; \mathrm{d} 244(\mathrm{P})$ & $(96,97 \mathrm{th})$ & 66 \\
\hline $20,21,24 / 2(A)$ & $(2,3,4)$ & 2 & $52 / 9$ & 98 & 67 \\
\hline $24 / 1(\mathrm{~A})$ & 5 & $\overline{3}$ & $8 / 6$ & 99 & 68 \\
\hline $36 / 4$ & 6 & 4 & $9 / 1$ & 100 & 69 \\
\hline $\mathrm{d} 289(\mathrm{P})$ & 7 & 5 & 49 & 101 & 70 \\
\hline $8 / 11$ & 8 & 6 & $27 / 6$ & 102 & 71 \\
\hline $47 / 1,47 / 4$ & $(9,10)$ & 7 & $10 / 4$ & 103 & 72 \\
\hline $22 / 5,26 / 4,36 / 3$ & $(11,12,13)$ & $\dot{8}$ & $5 / 3$ & 104 & 73 \\
\hline $10 / 3$ & 14 & 9 & $4 / 3,12 / 4,38$ & $(105,106,107)$ & 74 \\
\hline $36 / 1$ & 15 & 10 & $52 / 6$ & 108 & 75 \\
\hline $22 / 6$ & 16 & 11 & $40 / 2$ & 109 & 76 \\
\hline $22 / 1,22 / 4,36 / 2$ & $(17,18,19)$ & 12 & $32,34,43 / 2,43 / 3$ & $(110-114)$ & 77 \\
\hline $47 / 3,47 / 5$ & $(20,21)$ & 13 & KBI & & \\
\hline $47 / 2$ & 22 & 14 & d251 (P) & 115 & 78 \\
\hline $52 / 1,52 / 5 ; \mathrm{d} 52(\mathrm{P})$ & $(23,24,25)$ & 15 & $53 / 1$ & 116 & 79 \\
\hline 46 & 26 & 16 & 41 & 117 & 80 \\
\hline $4 / 2,15 / 6$ & $(27,28)$ & 17 & $5 / 10$ & 118 & 81 \\
\hline $52 / 4$ & 29 & 18 & $14 / 1,27 / 2$ & $(119,120)$ & 82 \\
\hline $35 / 1$ & 30 & 19 & $56 / 1$ & 121 & 83 \\
\hline $8 / 3,8 / 14,26 / 5,50$ & $(31-34)$ & 20 & $12 / 5,27 / 5$ & $(122,123)$ & 84 \\
\hline d53 (P) & 35 & 21 & $5 / 5$ & 124 & 85 \\
\hline $26 / 3,54$ & $(36,37)$ & 22 & $52 / 10$ & 125 & 86 \\
\hline $8 / 4$ & 38 & 23 & $27 / 3$ & 126 & 87 \\
\hline $13 / 3$ & 40 & 24 & $15 / 4$ & 127 & 88 \\
\hline $31 / 3$ & 41 & 25 & $19^{1}$ & 128 & 89 \\
\hline $56 / 3$ & 42 & 26 & $5 / 2$ & 129 & 90 \\
\hline $12 / 1,12 / 6$ & $(43,44)$ & 27 & $9 / 3$ & 130 & 91 \\
\hline $26 / 2,45$ & $(45,46)$ & 28 & $56 / 4$ & 131 & 92 \\
\hline $28 / 6,52 / 2$ & $(47,48)$ & 29 & $\mathrm{~d} 152(\mathrm{P})$ & 132 & 93 \\
\hline $7 / 3,8 / 2,8 / 15$ & $(49-52)$ & 30 & $10 / 1$ & 133 & 94 \\
\hline d263 (P) & & & $7810(\mathrm{Ae})$ & 134 & 95 \\
\hline $8 / 5,8 / 7,8 / 13,26 / 1$ & $(53-57)$ & 31 & $13 / 1$ & 135 & 96 \\
\hline $28 / 4$ & & & $15 / 5$ & 136 & 97 \\
\hline $8 / 9$ & 58 & 32 & $27 / 4$ & 137 & 98 \\
\hline 51 & 59 & 33 & $9 / 4$ & 138 & 99 \\
\hline $52 / 8$ & 60 & 34 & $43 / 1$ & 139 & 100 \\
\hline $8 / 12$ & 61 & 35 & $12 / 3$ & 140 & 101 \\
\hline $23 / 3$ & 62 & 36 & $31 / 1,31 / 2$ & $(141,142)$ & 102 \\
\hline $28 / 3 ; 28 / 5(\uparrow)$ & $(63,64)$ & 37 & $5 / 8$ & 143 & 103 \\
\hline $8 / 16$ & 65 & 38 & $56 / 2$ & 144 & 104 \\
\hline $27 / 1$ & 66 & 39 & $18 / 3$ & 145 & 105 \\
\hline $53 / 2$ & 67 & 40 & $40 / 3$ & 146 & 106 \\
\hline $8 / 8$ & 68 & 41 & $15 / 1$ & 147 & 107 \\
\hline $22 / 3$ & 69 & 42 & 44 & 148 & 108 \\
\hline $7357(\mathrm{C})$ & 70 & 43 & $43 / 4$ & 149 & 109 \\
\hline 8049 (Ae) & 71 & 44 & $13 / 4$ & 150 & 110 \\
\hline $23 / 4$ & 72 & 45 & $42 / 3$ & 151 & 111 \\
\hline $52 / 3$ & 73 & 46 & $9 / 2$ & 152 & 112 \\
\hline $7 / 6$ & 74 & 47 & $23 / 1$ & 153 & 113 \\
\hline $5 / 1$ & 75 & 48 & $\mathrm{~d} 221(\mathrm{P})$ & 154 & 114 \\
\hline $16 / 1$ & 76 & 49 & $18 / 2$ & 155 & 115 \\
\hline $8 / 10$ & 77 & 50 & $42 / 2$ & 156 & 116 \\
\hline 30 & 78 & 51 & $\mathrm{~d} 236(\mathrm{P})$ & 157 & 117 \\
\hline $42 / 1$ & 79 & 52 & d281 (P) & 158 & 118 \\
\hline $23 / 5$ & 80 & 53 & d270 (P) & 159 & 119 \\
\hline $5 / 4$ & 81 & 54 & $3246(\mathrm{~S})$ & 160 & 120 \\
\hline $15 / 3$ & 82 & 55 & $\mathrm{~d} 133$ (P) & 161 & 121 \\
\hline 55 & 83 & 56 & $25 / 2(X)$ & 162 & 122 \\
\hline $26 / 6$ & 84 & 57 & $\mathrm{~d} 248(\mathrm{P})$ & 163 & 123 \\
\hline 3 & 85 & 58 & $\mathrm{~d} 72(\mathrm{P})$ & 164 & 124 \\
\hline 39 & 86 & 59 & $\mathrm{~d} 260(\mathrm{P})$ & 165 & 125 \\
\hline $22 / 2,22 / 7$ & $(87,88)$ & 60 & $\mathrm{~d} 300(\mathrm{P})$ & 166 & 126 \\
\hline $16 / 2$ & 89 & 61 & d 69 (P) & 167 & 127 \\
\hline $13 / 2,14 / 2$ & $(90,91)$ & 62 & d 64 (P) & 168 & 128 \\
\hline $40 / 1$ & 92 & 63 & d $73(N)$ & 169 & 129 \\
\hline $35 / 2,35 / 3$ & $(93,94)$ & 64 & $8 / 1$ & 39 & 130 \\
\hline $52 / 7$ & 95 & 65 & & & \\
\hline
\end{tabular}


as used by Sneath $(1957 b)$, where $n_{s}$ is the number of positive features possessed by both isolates, and $n_{d}$ is the number of positive features possessed by the first isolate but not by the second + the number of features possessed by the second isolate but not by the first.

A sorting of the isolates into groups at 75.0, 80.0, 85.0, 90.0 and 95.0\% similarity levels of association ( $\mathrm{L}$ levels) was also made by the computer. At the $75 \cdot 0 \%$ level only 16/130 isolates remained ungrouped; these were sorted by visual inspection of the $\mathbf{S}$ table. Then family trees were constructed in an attempt to show the interrelationships revealed by the analysis.

\section{RESULTS AND DISCUSSION}

The order of the arrangement of isolates made by the computer was compared with the order which resulted when the numerical principles described above were used. Figure 1 illustrates a section of the experimental data and Table 2 gives a sample of the arrangements of the author (Rhodes, 1959) and the computer. In the sequel, C. no. = computer code number. The following conclusions may be drawn.

(a) The two arrangements of the isolates were often in close agreement (see Table 2).

Table 2. A comparison of the arrangements of the 39 most reactive isolates of Pseudomonas as made by the author and the computer

C. no. = Computer code number. $\dagger=$ proposed neotype strain of Pseudomonas fluorescens (NCTC no. 10,038; ATCC no. 13,525) selected by Rhodes (1959). * = isolates for which the author's placement and the computer's placement differed by more than 10 places.

\begin{tabular}{|c|c|c|c|c|c|}
\hline \multirow{2}{*}{$\begin{array}{c}\text { Position in } \\
\text { author's } \\
\text { table } \neq\end{array}$} & \multicolumn{2}{|c|}{$\begin{array}{l}\text { Order of isolates as } \\
\text { arranged by the }\end{array}$} & \multirow{2}{*}{$\begin{array}{l}\text { Position in } \\
\text { author's } \\
\text { table }\end{array}$} & \multicolumn{2}{|c|}{$\begin{array}{l}\text { Order of isolates as } \\
\text { arranged by the }\end{array}$} \\
\hline & $\begin{array}{l}\text { Author } \\
\text { (C. no.) }\end{array}$ & $\begin{array}{l}\text { Computer } \\
\text { (C. no.) }\end{array}$ & & $\begin{array}{l}\text { Author } \\
\text { (C. no.) }\end{array}$ & $\begin{array}{c}\text { Computer } \\
\text { (C. no.) }\end{array}$ \\
\hline 1 & 1 & 1 & 21 & 21 & 25 \\
\hline 2 & 2 & 2 & 22 & 22 & 26 \\
\hline $\mathbf{3}$ & $\mathbf{3}$ & $\mathbf{3}$ & 23 & $23^{*}$ & 28 \\
\hline 4 & 4 & 4 & 24 & $130^{*}$ & 13 \\
\hline 5 & 5 & 6 & 25 & 24 & 14 \\
\hline 6 & 6 & 10 & 26 & 25 & 29 \\
\hline 7 & 7 & 11 & 27 & 26 & 30 \\
\hline 8 & 8 & 12 & 28 & 27 & 31 \\
\hline 9 & $\boldsymbol{9}$ & 5 & 29 & 28 & 32 \\
\hline 10 & 10 & 7 & $\mathbf{3 0}$ & 29 & $\mathbf{3 4}$ \\
\hline 11 & 11 & 8 & 31 & $\mathbf{3 0}$ & $\mathbf{3 5}$ \\
\hline 12 & 12 & 9 & 32 & 31 & 27 \\
\hline 13 & $13^{*}$ & 15 & $\mathbf{3 3}$ & 32 & 43 \\
\hline 14 & $14^{*}$ & 16 & 34 & 33* $^{*}$ & 18 \\
\hline 15 & 15 & 17 & $\mathbf{3 5}$ & 34 & $\mathbf{2 3}$ \\
\hline 16 & 16 & 19 & 36 & 35 & 130 \\
\hline 17 & 17 & 20 & 37 & $36^{*}$ & 38 \\
\hline 18 & $18^{*}$ & 21 & 38 & $37 \dagger$ & 39 \\
\hline 19 & 19 & 22 & 39 & 38 & $37 \dagger$ \\
\hline 20 & 20 & 24 & & & \\
\hline
\end{tabular}


(b) Of the 7 isolates illustrated in Table 2 which showed positions differing by more than 10 places, for 5 of them (C. no. 13, 14, 18, 23, 130) this was apparently due to the author's failure to give sufficient weight to an isolate which possessed a relatively rare character (aesculin hydrolysis). For example, isolate C. no. 23 which was aesculin positive and had only 7 negative characters was computed to be 12 places later in the sequence and after isolate C. no. 35 which had 8 negative characters. Similarly, isolate C. no. 36 with two relatively rare positive characters (growth at $42^{\circ}$; ability to produce $\mathrm{H}_{2} \mathrm{~S}$ from cystine) was placed much later (in the 77th position) by the computer. Likewise isolate C. no. 33 which possessed three relatively rare positive characters and only 8 negative ones was given a position 14. places later by the computer than by the author, and then came after isolates with 9 or even 10 negative characters. Therefore the fact that my placements which were too near the 'reactive' beginning of my table in comparison with the order given by the computer, suggests that the possession of even one relatively rare positive character did not receive sufficient emphasis as a dissimilarity when a comparison was made on my numerical basis. In other words, the formula used by Sneath $(1957 b)$ for the electronic computation gave a better over-all comparison than was achieved solely by numerical counting of numbers of positive and negative characters. This was because when using the latter method relatively rare positive characters were not sufficiently weighted as dissimilarities.

(c) The case of isolate C. no. 130 was consoling. This isolate was placed after isolate C. no. 23 in the author's original table (see Fig. 1; Tables 1 and 2) but in the preparation of the data for the computer it was accidentally omitted and added to the list as isolate C. no. 130 (see Table 1). The computer placed it back to follow isolate C. no. 23 once more.

(d) The only other gross aberrations in the comparison concerned isolate NCTC 8049 which was placed 44th by the author and 108th by the computer, and isolate NCTC7810 which was placed 95th and 109th respectively. These isolates were the two aeromonads already differentiated from the rest of the collection by other criteria (Rhodes, 1959). They were included here to test the electronic computation method which indeed placed them together, even though one had only 9 and the other 15 negative characters. They were positioned by the computer between the Pseudomonas fluorescens-like isolates and the phytopathogens (see Ae, Fig. 2); but this positioning is misleading because the characters used to differentiate the genus Aeromonas were not among those considered in the present work (see later). Another non-Pseudomonas isolate (C. no. 129) was clearly differentiated from the rest by the computer (see Fig. 2) at the $15.0 \%$ level; the one xanthomonad was grouped with two other phytopathogens at the $45.0 \%$ level.

The $S$ table was converted to a shaded diagram as suggested by Sneath (1957b). It was evident that the isolates down to C. no. 110 formed a large group with $\mathbf{8 0 . 0} \%$ over-all similarity throughout. The sorting procedure revealed that within this large group some subgroups with a higher $\%$ over-all similarity were present. These are shown more clearly in Fig. 2 than in the shaded diagram; it was not considered worth while to re-arrange the $S$ table because of the high over-all similarity of all the isolates in the large group. The shaded diagram proved too large and complex to reproduce here; it has been photographed and copies are available. 


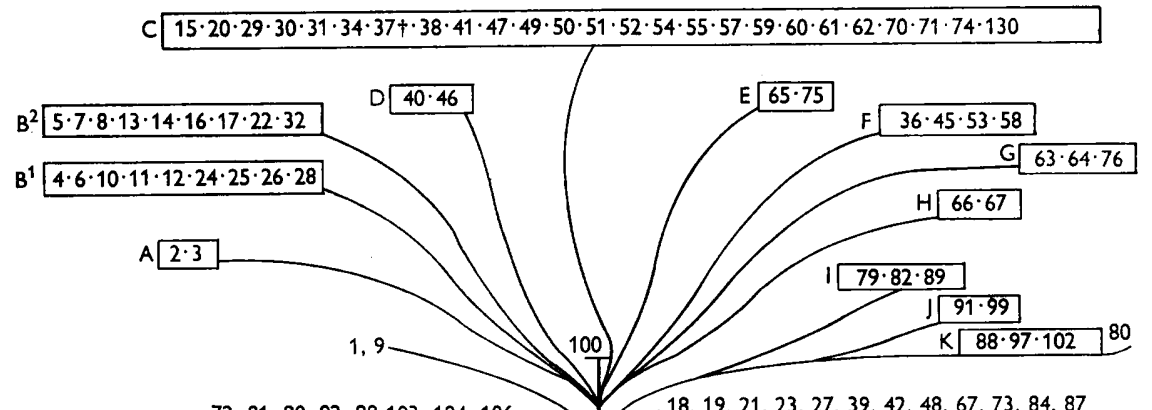

$72,81,90,92,98,103,104,106$

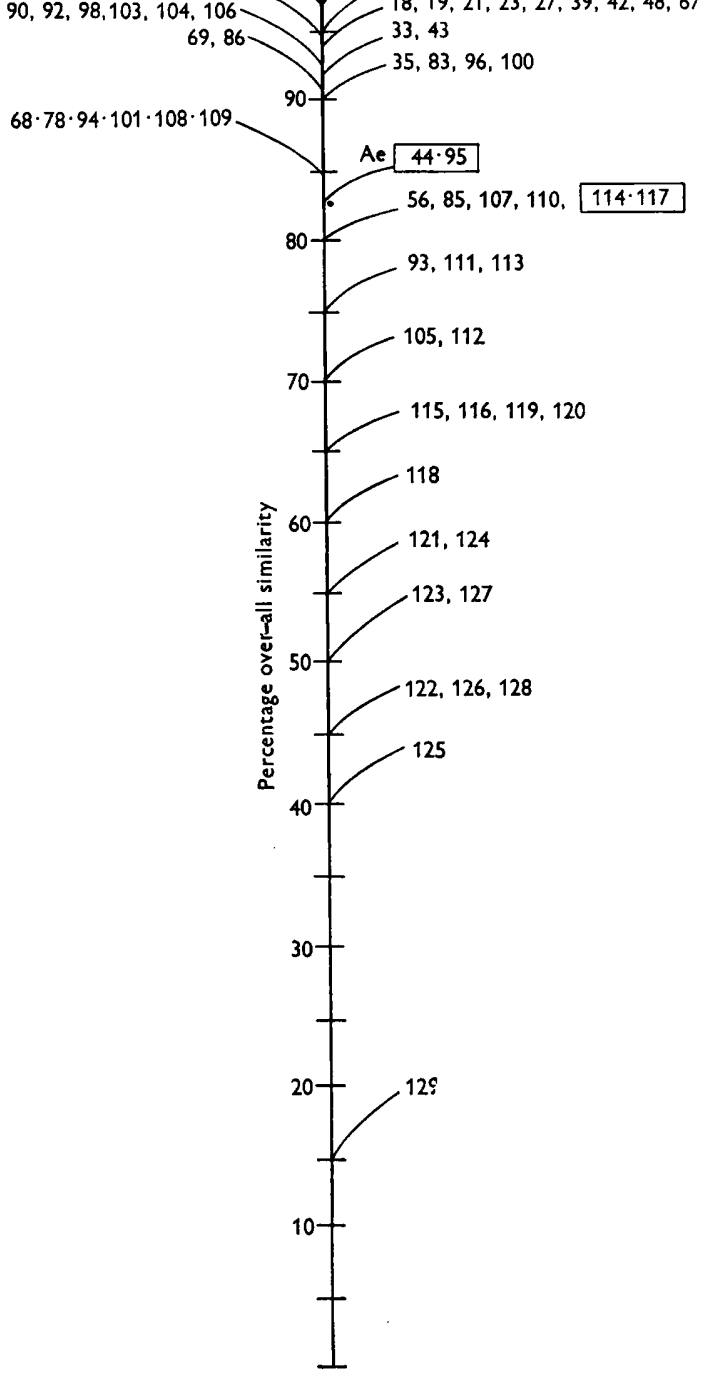

Fig. 2. Diagram showing the inter-relationships between 130 isolates, mainly Pseudomonas, constructed from the $S$ table of over-all similarites and sorted by the electronic computer on the basis of the 45 test characters listed in Fig. 1.

Note that further isolates may be included in some of the groups, i.e. replicates listed in Table 1. Boxed-in groups of isolate numbers, indicated by letters, contain isolates with more than $97.0 \%$ over-all similarity among themselves. Numbers indicate the computer no. (C. no.) of the isolate. $\dagger=$ proposed neotype strain of $P$. fluorescens (Rhodes, 1959); group $\mathbf{A}=P$. aeruginosa isolates; groups $B$ to $K$ together with isolates down to 110 (at $80.0 \% \mathrm{~S}$ level) $=\boldsymbol{P}$. fluorescens species-group; between 40.0 and $\mathbf{7 0 . 0} \% \mathrm{~S}$ values = phytopathogenic Pspudomonas species; group Ae = Aeromonas isolates (but see text about 'true' position) $122=$ ? Xanthomonas sp.; $129=$ non-pseudomonad phytopathogen. 
Thus the analysis by the electronic computer confirmed the previous conclusions of the author about the recognition of the 134 soil- and water-inhabiting pseudomonads as a group of closely related organisms. It also confirmed that the description of the species Pseudomonas fluorescens yielded a useful taxonomic unit, even though there were no absolute correlations between any two of the descriptive characters. Indeed, many of the characters were often absent from the isolates examined. This large group was known to contain isolates of $\boldsymbol{P}$. aeruginosa and

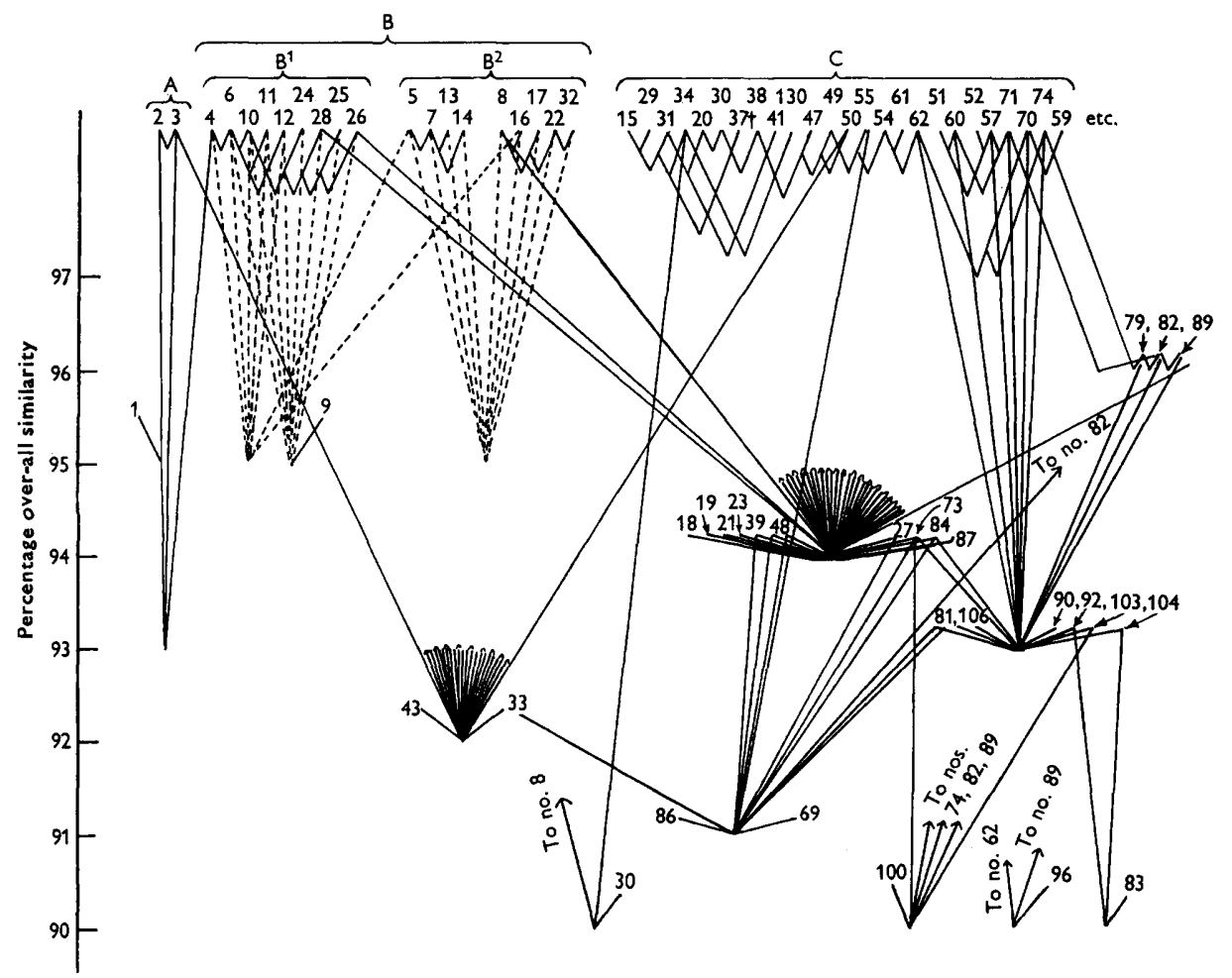

Fig. 3. Diagram showing the inter-relationships between many isolates of Pseudomonas when their over-all similarity was assessed on the basis of 45 test characters (listed in Fig. 1). The lines join isolates together at various levels of over-all similarity. It is apparent that a given isolate may show an equally close similarity with members in various groups, thus exhibiting multiple relationships. The dotted lines are used merely for clarity and are not otherwise significant. All numbers (some not included owing to the mechanical difficulties of reproduction) refer to the computer number (C. no.) of the isolate. $\dagger=$ proposed neotype strain of Pseudomonas fluorescens (Rhodes, 1959).

five named phytopathogenic species from the National Collection of Type Cultures. The close similarity of these with $\boldsymbol{P}$. fluorescens has already been discussed (Rhodes, 1959). It may be relevant that the phytopathogens examined had been maintained in laboratory culture for a long time, and at least one of them (d52, P. medicaginis var. phaseolicola) when examined was found to be identical with two non-phytopathogenic isolates (see Table 1) and did not produce the characteristic greasy spots when inoculated into young bean pods. Nor was it susceptible to active $\boldsymbol{P}$. medicaginis var. phaseolicola bacteriophage (Dr Eve Billing, personal communication). 


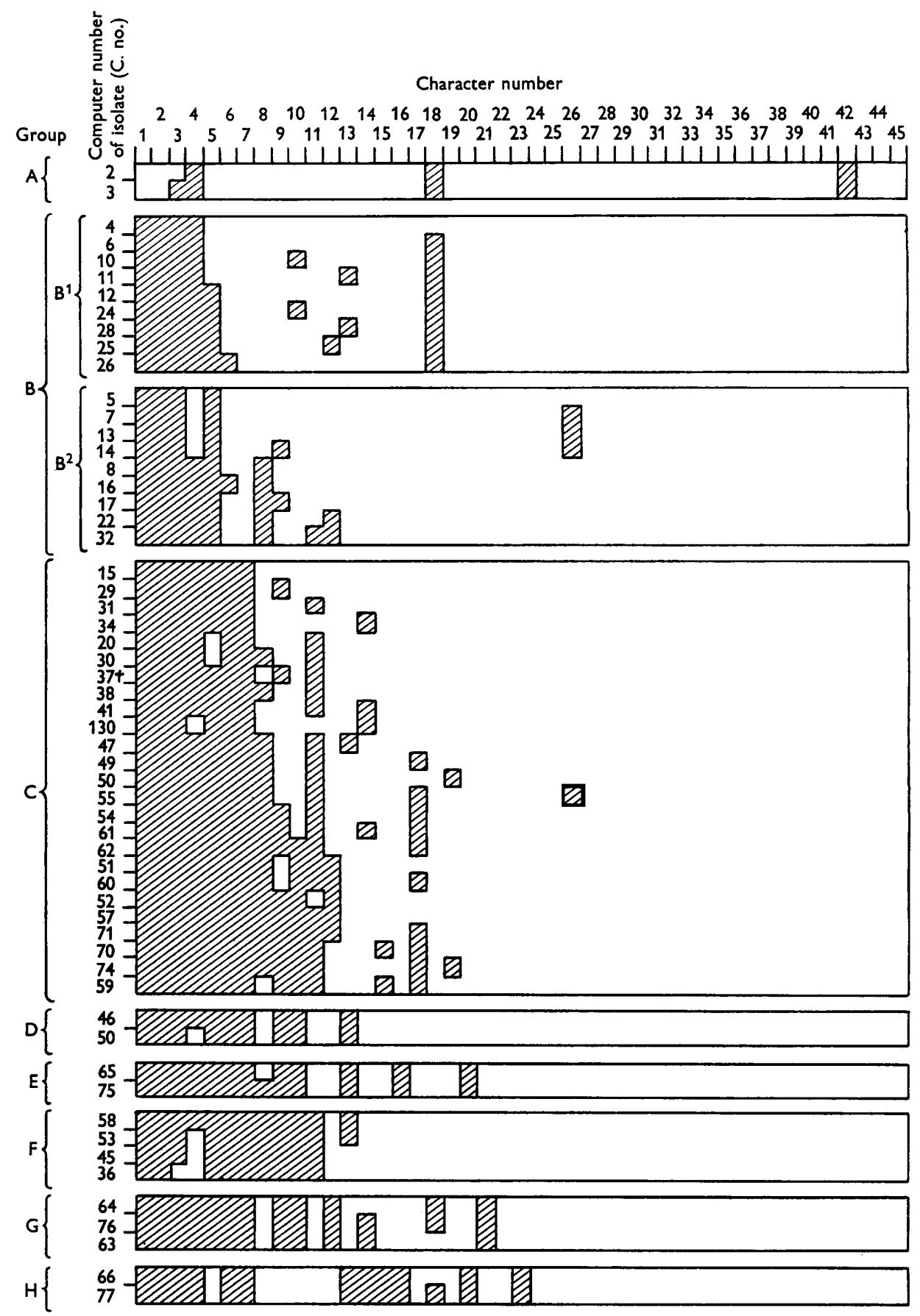

Fig. 4. For legend see opposite. 
Figure 3 is another diagrammatic method for showing some of the more detailed relationships revealed by the $\mathrm{S}$ table; it attempts to show the multiple and overlapping relationships between some of the isolates. It can be seen that some groups (labelled A, B, C, etc., in the diagrams) of more closely similar isolates could be differentiated within the large Pseudomonas fluorescens group. The question whether such subgroups merit the rank of species, subspecies or variety is a major problem of bacterial taxonomy (see discussion by Sneath, 1957b, pp. 212-216). If one wishes to retain $\boldsymbol{P}$. aeruginosa as a species then, according to this analysis, this means that all the aeruginosa-like isolates (group $\mathbf{A}$ here) should have an overall similarity of more than about $95.0 \%$, because at the $93.0 \%$ level group $\mathbf{A}$ (aeruginosa-type) and group B (fluorescens-type) are linked by isolates such as C. no. 4 (see Fig. 3) which is definitely fluorescens-like. Furthermore, at the $\mathbf{9 2 . 0} \%$ level aeruginosa-like isolate C. no. 3 has a similarity of $\mathbf{9 2 . 0} \%$ or more with at least 30 fluorescens-type isolates. Therefore if group $\mathbf{A}$ were to be regarded as a species (P. aeruginosa), then by the same standards (based on $\mathbf{S}$ values) groups $\mathbf{B}, \mathbf{C}$, D, E, F, G and $\mathbf{H}$ (see Figs. 2-4) should also be considered as species. Whether group $\mathbf{B}$ is itself composed of $\mathbf{2}$ or $\mathbf{3}$ species, i.e. groups $\mathbf{B}^{\mathbf{1}}$ and $\mathbf{B}^{\mathbf{2}}$ (see Figs. 2-4) is also debatable. To help the crystallization of opinion Fig. 4 is given to show the familiar bacteriological properties of each of these groups and the range of variation exhibited. It is apparent that black and white definitions of such groups (? species) are not possible. In the author's opinion not one of these groups merits species rank; they are better to be regarded as units of lower taxonomic rank, e.g. varieties of $\boldsymbol{P}$. fiuorescens, even though the group $\mathbf{A}$ isolates are typical $\boldsymbol{P}$. aeruginosa. This conclusion is, of course, controversial. It raises taxonomic and nomenclatural difficulties, because $\boldsymbol{P}$. aeruginosa was designated as the type species of the conserved genus Pseudomonas by the Judicial Commission of the International Committee on Bacteriological Nomenclature (1952). Nevertheless, it confirms the practical experiences of many Pseudomonas taxonomists (see Haynes, 1951; Gaby \& Free, 1953; Gaby \& Hadley, 1957) about the close similarity of $\boldsymbol{P}$. aeruginosa and $\boldsymbol{P}$.

Fig. 4. The bacteriological properties of isolates of Pseudomonas which had been grouped together by the electronic computer at an over-all similarity level of more than $97.0 \%$ on the basis of 45 test characters (listed below). Groups are indicated by capital letters and are the same as the groups shown in Fig. 2. Hatched areas indicate negative test results; clear areas indicate positive test results. $\dagger=$ proposed neotype strain of Pseudomonas fluorescens (Rhodes, 1959). Key to character numbers: $1=\mathrm{H}_{2} \mathrm{~S}$ from Kligler's medium; $2=$ growth at $42^{\circ} ; 3=\mathrm{H}_{2} \mathrm{~S}$ from cystine; $4=$ aesculin hydrolysis; $5=$ growth in $6.5 \% \mathrm{NaCl}$-broth; $6=$ nitrite reduction; $7=$ nitrate reduction $; 8=$ growth from raffinose- $\mathrm{C} ; \mathbf{9}=$ growth in minus-C control; $10=$ growth in minus- $\mathrm{N}$ control; $11=$ growth in urea-N; $12=$ growth from sucrose-C; $13=$ hydrolysis of 'Tween-80'; $14=$ milk digestion; $15=$ egg-yolk reaction; $16=$ olive oil hydrolysis; $17=$ growth from inositol-C; $18=\mathrm{H}_{2} \mathrm{~S}$ from thiosulphate; $19=$ growth from cellobiose-C; $20=$ tributyrin hydrolysis; $21=$ gluconic acid oxidation; $22=$ growth from trehalose-C; $23=$ gelatin liquefaction; $24=$ growth from rhamnose- $\mathrm{C} ; 25=$ growth in bile-salt broth; $26=$ growth from D-arabinose-C; $27=$ growth in $5.0 \%$ NaCl-broth; $28=$ growth from succinate-C; 29 = growth from acetate-C; $30=$ growth from mannitol-C; $31=$ growth from lactic acid-C; $32=$ growth from lactose-C; $33=$ growth from glycerol-C; 34 = growth from xylose-C; 35 = growth from formate-C; 36 = growth from L-arabinose-C; $37=$ growth from maltose-C; $38=$ growth at $\mathrm{pH} 5 \cdot 0 ; 39=$ growth from glucose-C; $40=$ growth from galactose-C; $41=$ growth at $5^{\circ} ; 42=$ growth from fructose-C; 43 = growth from 1 -malic acid-C; $44=$ growth from citrate-C; $45=$ growth from sorbitol-C. 
fluorescens. The difficulties encountered when attempts were made to define the 'highly dissociable' genotype species $\boldsymbol{P}$. aeruginosa were described by Gaby (1955) who found it impossible to designate a type culture; no exact definition of it is available. The analogous situation with the anthrax bacillus may be mentioned and particularly the reasoning of Smith, Gordon \& Clark (1952) which led to their conclusion that the anthrax bacillus was best to be regarded as a potentially pathogenic variety of Bacillus cereus. Evidence from the literature and practical experience support the hypothesis that $\boldsymbol{P}$. aeruginosa may be a variety derived from a fluorescenslike wild-type organism as a result of adaptation to the specialized environment of the animal body with which it is usually associated directly or indirectly. $\boldsymbol{P}$. aeruginosa seldom occurs elsewhere, perhaps because it cannot withstand a temperature of less than $5^{\circ}$ for more than 3 months (when stored in nutrient broth culture; personal observation). Lethal cold-shocking of $\boldsymbol{P}$. aeruginosa was recently described by Gorrill \& McNeil (1960). Finally, in connexion with the question of species rank, the convincing Chromobacterium subgroups which were designated as species by Sneath $(1957 b)$ appeared as separate groups down to an $S$ value of $64.0 \%$; his $\%$ values are perhaps comparable with mine because the experimental criteria from which they were derived were similar.

The position of the two Aeromonas isolates and their apparent fusion with the Pseudomonas fluorescens group at the $83.0 \% \mathrm{~S}$ level (see Fig. 2) is misleading because their distinctly different non-pseudomonad properties, which relate to their characteristic fermentative metabolism, were characters not included in the present analysis. A comparison was made which included all the typical aeromonad properties (see Rhodes, 1959, p. 248) as well as those used in this analysis. The $\mathrm{S}$ level values were then $\mathbf{5 8 . 0}$ and $\mathbf{4 5 . 0} \%$ for Aeromonas icthyosmia and A. hydrophila, respectively, when they were compared with the proposed neotype strain of $P$. fluorescens.

The 19 isolates at the lowest part of Fig. 2 were different from the rest of the collection, and in most cases also differed from each other. This agrees with the fact that they consisted of representatives of at least 11 named phytopathogens, Pseudomonas syncyanea and 2 non-Pseudomonas isolates. This suggests that differences which merit species rank may be revealed when larger numbers of phytopathogenic isolates are similarly studied.

Another method of demonstrating some of the $\mathrm{S}$ table data was suggested by Cheeseman \& Berridge (1959). This involved the arrangement of the isolates in a histogram which showed the numbers of $S$ values of more than $70.0 \%$ possessed by each isolate. This method was examined in the present work. The results are given in Fig. 5 (solid lines). The order given by this treatment of the data did not follow the computer order at all closely, except for the placement of the same last 25 isolates. Those isolates with a large number of high $\mathrm{S}$ values were presumed to be most closely interrelated and therefore most typical of the whole group (Cheeseman \& Berridge, 1959). Such reasoning implies that the first isolate here, C. no. 4, with $82 \mathrm{~S}$ values of $70.0 \%$ would be a better neotype strain of Pseudomonas fluorescens than the isolate C. no. 37 previously selected by the author (deposited in the NCTC as No. 10,038 and in the American Type Culture Collection, ATCC, as No. 13,525). It was felt by the author (Rhodes, 1959) that the most useful type strain of a group of organisms showing a given range of + ve or - ve characters should occur centrally 
within this range, as indeed C. no. 37 does. Isolate C. no. 4 is considered by me to be unusually reactive. I think that in practice the likelihood that an unidentified new $\boldsymbol{P}$. fluorescens-like isolate would be closely similar to such a type strain would probably be less than if a mediumly reactive isolate such as isolate C. no. 37 were selected as type strain.

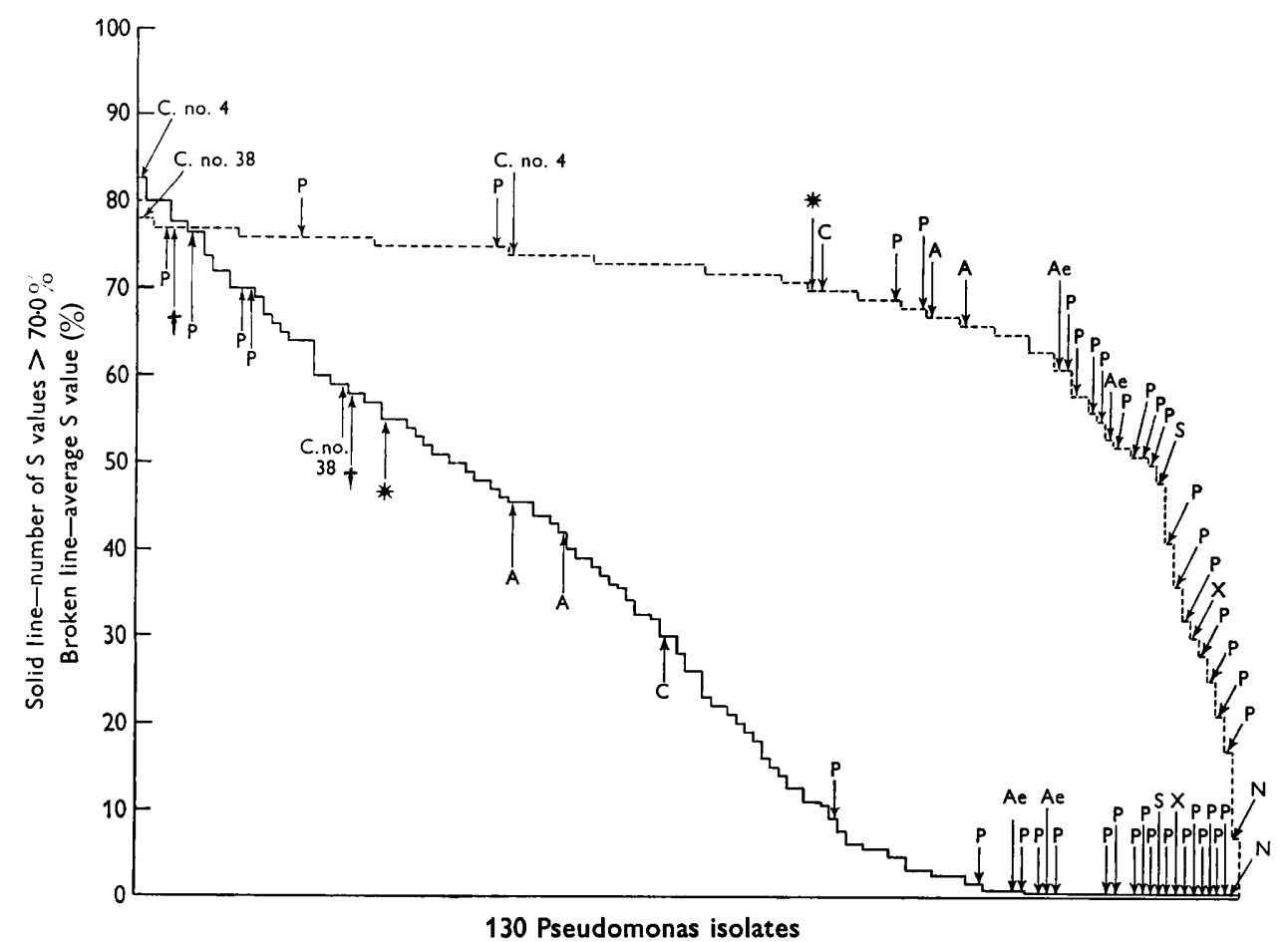

Fig. 5. Histograms showing the numbers of $S$ values of more than $70.0 \%$ possessed by each of 130 isolates of (mainly) Pseudomonas (solid lines), and also the average $S$ values of each isolate (broken lines). It is not possible to include the code numbers of each isolate in the diagrams, but some of the isolates discussed are shown by letters, the key for which is : $\dagger=$ proposed neotype strain Pseudomonas fuorescens (Rhodes, 1959); $*=$ NCTC $P$. fluorescens $; \mathrm{C}=P$. chlororaphis $; \mathrm{A}=P$. aeruginosa $; \mathrm{S}=P$. syncyanea $;$ $\mathbf{P}=$ phytopathogenic Pseudomonas sp.; Ae = Aeromonas sp.; $\mathbf{X}=$ Xanthomonas sp.; $\mathbf{N}=$ non-pseudomonad phytopathogen; C. no. $4=$ computer number $4 P$. fluorescens isolate $36 / 4$; C. no. $38=$ computer number 38 P. fluorescens isolate 8/16.

I am grateful to Dr P. H. A. Sneath for pointing out that for a given isolate, average $S$ values rather than numbers of $S$ values of greater than $70.0 \%$ are perhaps better criteria for isolate comparison. Following this suggestion, the average $\mathbf{S}$ values for each of the 130 'computer' isolates were calculated by adding together all the appropriate rows and columns of numbers in the $\mathbf{S}$ table and dividing the total for each isolate by 129 (i.e. ignoring the $100.0 \%$ value of self-comparison). The results are shown in Fig. 5 (broken lines). The highest average $\mathrm{S}$ value was $78.11 \%$, given by isolate C. no. 38; isolate C. no. 4 (discussed above) was relegated to the 45th position. In all the diagrams it is evident that isolate $\mathrm{C}$. no. 38 is very similar to the proposed (Rhodes, 1959) neotype strain Pseudomonas fluorescens (isolate C. no. $\mathbf{3 7}$ here). Isolate C. no. $\mathbf{3 7}$ also has the 5 th highest average $\mathbf{S}$ value 
$(\mathbf{7 7} \cdot \mathbf{8 4} \%$ ). Therefore the suitability of isolate C. no. 37 to be the type strain of $\boldsymbol{P}$. fluorescens is confirmed; it fulfils the requirements of a 'type culture' of a bacterial species as defined in Bergey's Manual (1957, p. 21) namely: 'A species of bacterium is the type culture or specimen together with all the other cultures or specimens regarded by an investigator to be sufficiently like the type (or sufficiently closely related to it) to be grouped with it.'

An inspection of the average $S$ values of each isolate of the collection examined here showed a group of 152 isolates (including replicates; see Table 1) with average $\mathrm{S}$ values between $\mathbf{6 3 . 0}$ and $\mathbf{7 8 . 0} \%$. The whole group included 7 phytopathogenic species of Pseudomonas, $\boldsymbol{P}$. chlororaphis, 5 isolates of $\boldsymbol{P}$. aeruginosa, 2 aeromonads, and 137 fluorescens-type isolates. Of these latter 137 isolates, 134 were exactly the same soil- and water-type isolates as were used for the suggested (Rhodes, 1959) revised description of $\boldsymbol{P}$. fluorescens Migula, 1894. It is apparent that the analysis by the computer has confirmed many of the views held by the author (Rhodes, 1959) about the complex inter-relationships between pseudomonad isolates even when these were obtained only after the operation of the considerable selective factors inherent in the methods of isolation (e.g. one temperature, one medium, only fluorescent colonies selected), maintenance and study. The advantages of the judicious use of mechanical aids, such as an electronic computer in the analysis of data are obvious; but the tedium involved in the acquisition of sufficiently large quantities of reliable data under standardized conditions has not yet been circumvented. However, the author feels that the use of the electronic computer facilitates some advance from the situation criticized by Rahn (1929) who wrote: 'The method of attacking taxonomical problems by securing a large number of strains is the most hopeful of all ways; but the treatment of the data has not always shown that a real distinction has been made between species and strains.'

I am much indebted to Dr P. H. A. Sneath (National Institute for Medical Research, Mill Hill, London) for his interest and assistance, and to the Research Board of the University of Reading for a grant enabling the hire of the computer. Elliott Brothers Ltd. (Boreham Wood, Hertfordshire, England) most generously processed all my data, and I wish to thank Mr G. P. Wayne for his services on behalf of the firm. The interest and help of Professor B. C. J. G. Knight in the preparation of this paper is also gratefully acknowledged.

\section{REFERENCES}

Bergey's Manual of Determinative Bacteriology (1957). 7th ed. Ed. by R. S. Breed, E. G. D. Murray \& N. R. Smith. London: Baillière, Tindall and Cox.

Cheeseman, G. C. \& Berridge, N. J. (1959). The differentiation of bacterial species by paper chromatography. VII. The use of electronic computation for the objective assessment of chromatographic results. J. appl. Bact. 22, 307.

GaBy, W. L. (1955). Taxonomic problems relating to the identification of species within the genus Pseudomonas. Int. Bull. bact. Nomencl. 5, 153.

GABY, W. L. \& FreE, E. (1953). Occurrence and identification of non-pigmented strains of Pseudomonas aeruginosa in the clinical laboratory. J. Bact. 65, 746.

Gaby, W. L. \& Hadley, C. (1957). Practical laboratory test for the identification of Pseudomonas aeruginosa. J. Bact. 74, 356.

Gorrill, R. H. \& MCNeIL, E. M. (1960). The effect of cold diluent on the viable count of Pseudomonas pyocyanea. J. gen. Microbiol. 22, 437. 
Haynes, W. C. (1951). Pseudomonas aeruginosa-its characterization and identification. J. gen. Microbiol. 5, 939.

International Bacteriological Code of Nomenclature (1952). Editorial Board. Opinion No. 5. Conservation of the generic name Pseudomonas Migula 1894 and designation of Pseudomonas aeruginosa (Schroeter) Migula 1900 as Type Species. Int. Bull. bact. Nomencl. $2,121$.

RAHN, O. (1929). Contributions to the classification of bacteria. Zbl. Bakt. (2 Abt.), $78,1$. Rhodes, M. E. (1956). The classification of Pseudomonas. Ph.D. Thesis, University of Reading.

Rhodes, M. E. (1959). The characterization of Pseudomonas fluorescens. J. gen. Microbiol. 21, 221.

Smith, N. R., Gordon, R. E. \& Clark, F. E. (1952). Aerobic sporeforming bacteria. U.S. Dep. Agric. Monogr. no. 16.

Sneath, P. H. A. (1956). Cultural and biochemical characteristics of the genus Chromobacterium. J. gen. Microbiol. 15, 70.

SNEATH, P. H. A. (1957a). Some thoughts on bacterial classification. J. gen. Microbiol. 17, 184.

SNEATH, P. H. A. (1957 b). The application of computers to taxonomy. J. gen. Microbiol. 17, 201.

White, P. B. (1937). Remarks on bacterial taxonomy. Zbl. Bakt. (2. Abt.), 96, 145. 